\title{
Simulation of Conduction Cooled SRF Cavity
}

\author{
Jessica Thompson ${ }^{1,2}$ and Ram Dhuley ${ }^{2}$
}

\begin{abstract}
SRF cavities are used heavily in high energy physics research, but the operational complexities of SRF cavities prevent their widespread use in industry. One notable complexity of operating an SRF cavity is liquid helium coolant, and replacing liquid helium with cryocooler conduction cooling is an important research and development interest. As part of this effort, a system consisting of a $650 \mathrm{MHz}$ single cell cavity, a commercial grade pulse tube refrigerator, and a thermal conduction link was simulated through finite element analysis. The electromagnetic fields inside the cavity, heat flow through the system, and temperature distribution of the thermal link and cavity were calculated. Based on the simulation, we found that a $2 \mathrm{~W}$ @ 4.2 $\mathrm{K}$ pulse tube refrigerator is capable of cooling a single cell $\mathrm{Nb}_{3} \mathrm{Sn}$ coated cavity operating at up to $12 \mathrm{MV} / \mathrm{m}$ accelerating gradient. The maximum simulated temperature of the cavity was below 7 $K$, and the thermal conduction link was shown to significantly attenuate the cryocooler thermal oscillations at the location of the cavity.
\end{abstract}

\section{INTRODUCTION}

Radio frequency (RF) cavities are a key accelerator technology in particle physics research, and their use extends into the industrial sector. RF cavities show great potential in applications across a variety of fields, including medical imaging, sterilization, water treatment, and electron beam welding [1].

Although RF cavities can be constructed from normal conducting materials like copper, superconducting RF (SRF) cavities have substantially lower power losses. SRF cavities must be kept at cryogenic temperatures and are usually immersed in liquid helium. However, liquid helium systems are complicated, expensive, and large, so cryogenic systems which do not require liquid helium are an attractive alternative for cooling SRF cavities.

The Department of Energy and Fermilab have funded a Laboratory Directed Research and Development (LDRD) project to demonstrate conduction cooling of an SRF cavity. This project aims to design a system for keeping an SRF cavity cold using a cryocooler instead of liquid helium.

\section{BACKGROUND}

\section{A. Basics of Superconductivity}

Superconductivity is an effect observed in some materials below a critical temperature, $T_{c}$. Superconductive materials experience no electrical resistance to dc current and exhibit the Meissner effect, in which small external magnetic fields are expelled from the material. However, superconductivity breaks down if the material is subjected to temperatures higher than $T_{c}$ or magnetic fields above a critical magnitude, $B_{c}$. The

\footnotetext{
${ }^{1}$ University of Alabama, Tuscaloosa, AL 35487

${ }^{2}$ Fermi National Accelerator Laboratory, Batavia, IL 60501
}

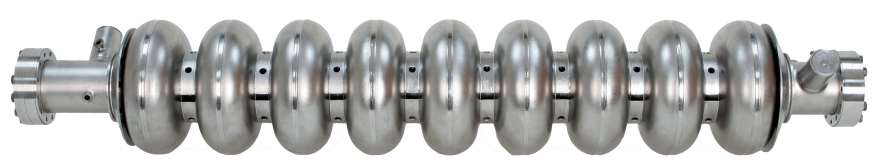

Fig. 1. A 9 Cell $1.3 \mathrm{GHz}$ Niobium Cavity [4].

maximum temperature, $T_{\max }$, and maximum surface magnetic field, $B_{p k}$, of an SRF cavity must remain below these limits. SRF cavities are usually formed from niobium, which has the highest critical temperature of any element at $9.25 \mathrm{~K}$ and a critical field value of $206 \mathrm{mT}$ at absolute zero temperature [2].

The Bardeen-Cooper-Schrieffer (BCS) Theory of Superconductivity describes superconductors as containing a mixture of normal electrons and Cooper pairs, pairs of electrons which can move freely through the metal lattice with no resistance [3]. When a superconductor is placed in a magnetic field which is weaker than $B_{c}$, a current of Cooper pairs is induced on the surface of the material. This current shields the normal electrons from the magnetic field, preventing resistive losses.

However, since Cooper pairs have mass, they cannot react instantaneously to changing magnetic fields. In ac fields, the Cooper pairs incompletely shield the normal electrons from the magnetic field, resulting in small resistive losses. The resistive losses of an SRF cavity can be quantified by the surface resistance $R_{s}$, which increases with temperature, frequency, and chemical impurities.

\section{B. SRF Cavity Fundamentals}

SRF cavities are hollow shells of metal which particles can pass through. Cavities are structured as elliptical cells with straight sections of beampipe in between, as shown in Figure 1. A cavity can have one or more cells.

SRF cavities are designed with a unique shape which resonates at a particular frequency. When a source supplies electric power at this frequency to the cavity, the electromagnetic waves inside the cavity build up through positive interference. The electric field points along the axis of the cavity and oscillates at the resonant frequency. Charged particles which travel through a cell when the electric field is strongest will see a high voltage across the cell and gain energy.

Several important parameters characterize the operation and efficiency of an SRF cavity, including accelerating gradient, dissipated power, quality factor, geometric factor, and normalized shunt impedance. In general, these parameters depend

This manuscript has been authored by Fermi Research Alliance, LLC under Contract No. DE-AC02-07CH11359 with the U.S. Department of Energy, Office of Science, Office of High Energy Physics. 
upon the cavity geometry and material, as well as on the driving source of RF power.

The accelerating gradient, $E_{a c c}$, of an SRF cavity measures the maximum energy gain per unit length. A particle traveling along the axis of a cavity cell will see a voltage difference across the cell, $V_{a c c}$. The accelerating gradient can be found by dividing by the length of the cell, $d$.

$$
E_{a c c}=\frac{V_{a c c}}{d}
$$

Since the RF field within the cavity varies with time, $V_{a c c}$ is dependent on the phase of the field when the particle enters the cavity and the speed of the particle moving through the cavity. To maximize the energy gain, particles must be carefully timed to reach the center of the cavity when the field is maximal.

Because a superconductor in an ac field has a nonzero surface resistance, an SRF cavity loses some energy in the form of heat. This dissipated power, $P_{d}$, is dependent on $R_{s}$ and on the magnetic field, $H$. With $\mathrm{S}$ as the inner surface of the cavity,

$$
P_{d}=\frac{1}{2} \int_{S} R_{s}|H|^{2} d A
$$

The quality factor, $Q_{0}$, of a cavity is the ratio of energy gained to power dissipated in one period. Quality factor varies with accelerating gradient. For a cavity with angular frequency $\omega$ and a total energy $U$,

$$
Q_{0}=\frac{\omega U}{P_{d}}
$$

The geometric factor, $G$, of an SRF cavity can be determined from only the geometry of the cavity and the eigenmode of operation. With $\mu_{0}$ as the permeability of free space,

$$
G=\frac{\mu_{0} \omega \int_{V}|H|^{2} d V}{\int_{S}|H|^{2} d A}
$$

The geometric factor defines a relationship between the quality factor and the surface resistance:

$$
G=Q_{0} R_{s}
$$

The normalized shunt impedance, $R / Q$, relates the voltage across the cavity to the stored energy.

$$
R / Q=\frac{V_{a c c}^{2}}{\omega U}
$$

If $R_{s}$ is assumed constant across the cavity, the dissipated power can be approximated by

$$
P_{d}=\frac{V_{a c c}^{2} R_{s}}{G(R / Q)}=\frac{d^{2} E_{a c c}^{2} R_{s}}{G(R / Q)}
$$

\section{Basics of Cryocoolers}

An increasing demand for very low temperature, or cryogenic, applications has led to a growing market of commercial grade cryogenic coolers, or cryocoolers. Tabletop cryocoolers with high heat capacity are widely available and can cool applications to $4 \mathrm{~K}$ and below.

A pulse tube refrigerator (PTR) is a type of cryocooler with no moving parts at low temperature, an advantage which

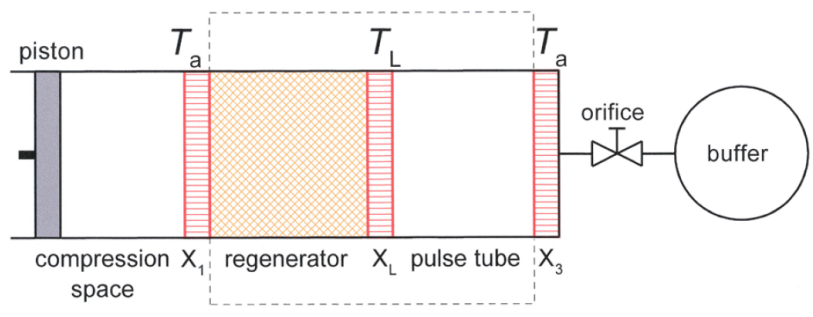

Fig. 2. Parts of a Typical Pulse Tube Refrigerator [7].

reduces mechanical maintenance and vibrations within the cryocooler [5]. Figure 2 shows the typical components of a pulse tube refrigerator. Here the dotted line surrounds the thermally insulated section of the cryocooler. Thermal insulation can be achieved using vacuum and multi-layer insulation, which minimize both convection and radiation of heat [6].

The pulse tube refrigerator is composed of a piston, several heat exchangers $\left(X_{1}, X_{L}\right.$, and $\left.X_{3}\right)$, a regenerator, a pulse tube, an orifice, and a buffer volume. The piston moves back and forth, pushing the gas in and out of the insulated system and causing pressure variations in the pulse tube, a tube of fixed volume filled with gas. As the pressure in the pulse tube varies, the temperature rises and falls. During the low pressure stage of the cryocooler cycle, gas at room temperature $T_{a}$ moves from the buffer volume, a relatively large volume of gas at essentially constant pressure, into the pulse tube. The flow of gas is limited by the orifice. At the same time, gas flows out of the left side of the pulse tube at a low temperature $T_{L}$, supplying the cooling power through the heat exchanger $X_{L}$. During the high pressure stage of the cycle, gas enters the pulse tube through the regenerator, a heat exchanger made from a solid porous material, and leaves through the orifice at a high temperature, removing heat from the system [7].

The entire cooling cycle takes about one second to complete. During each cycle, the temperature within the pulse tube fluctuates, causing periodic temperature variations in the cooled application. In practice, most PTRs are designed in a U-shape so that the low temperature section, or cold head, of the cryocooler is positioned at the bottom of the cooler rather than in the middle. Two-stage pulse tube refrigerators, in which the first stage pre-cools the second stage, can reach temperatures as low as $2.1 \mathrm{~K} \mathrm{[7].}$

\section{Conduction Cooling a Cavity}

Advances in technology and the development of materials with favorable properties have paved the way for cooling an SRF cavity using a cryocooler rather than liquid helium. The high critical temperature of niobium allows cavities to operate above liquid helium temperatures. Furthermore, coating SRF cavities with $\mathrm{Nb}_{3} \mathrm{Sn}$ has been shown to substantially reduce the surface resistance (and thus, the heat dissipation of the cavity). The less stringent cooling requirements of modern SRF cavities, in combination with the growing availability of high capacity cryocoolers, have made conduction cooling SRF cavities possible. A thermal link was constructed to connect an SRF cavity to a cryocooler and allow heat to flow. The link 
was designed to conduct heat well at cryogenic temperatures and in vacuum, maintain good thermal contact with both the cryocooler and cavity, and prevent the mechanical vibrations of the cryocooler from affecting the cavity.

\section{E. Finite Element Analysis}

Finite element analysis (FEA) is a technique for numerically approximating the solutions to partial differential equations. Systems of partial differential equations with known boundary conditions are common in physics calculations, including heat transfer and electromagnetic field problems. Finite element analysis finds numerical values for unknown fields by dividing a continuous geometry into small discrete volumes. This process is called meshing, and the divided geometry is a mesh. Numerical values for the unknown fields are approximated for each element in the mesh, and the continuous solution is found by combining the results from all of the elements.

COMSOL is a software which implements FEA to solve problems across a variety of physics domains. COMSOL physics interfaces can be combined to perform multiphysics simulations, which model interactions from more than one branch of physics. To model the conduction cooled cavity, both electromagnetic and thermodynamic effects must be considered.

\section{Project GoAls And Procedure}

To demonstrate conduction cooling of an SRF cavity, a Cryomech PT420 pulse tube refrigerator (shown in Figure 3) will be used to cool a single cell $650 \mathrm{MHz}$ SRF cavity with $\mathrm{Nb}_{3} \mathrm{Sn}$ coating. The system was simulated to calculate RF parameters such as $\omega, P_{d}$, and $B_{p k}$, to determine the temperature distribution across the cavity and cooling link, and to examine the effect of cryocooler temperature oscillations on the cavity temperature.

\section{A. Geometry}

The simulated geometry includes the SRF cavity, vacuum space inside the cavity, cryocooler plate, and thermal link, as shown in Figure 4. Figure 5 shows half of the total geometry with the parts color coded.

Heat is generated on the inner surface of the cavity, and it flows through the thermal link to the cryocooler plate. To establish good thermal contact between the cavity and the cooling link, niobium rings are welded around the equator of the cavity. These rings are then bolted to the cooling link, providing a path for heat to flow out of the cavity. The thermal link is attached to the cryocooler plate with a U-shaped bend, which provides flexibility to isolate the mechanical vibrations of the cryocooler from the cavity. The entire system is kept under vacuum and is insulated with multi-layer insulation.

\section{B. Material Properties}

Several material-specific properties are needed to simulate heat transfer across the cavity and cooling link, including thermal conductivity, specific heat capacity, and thermal contact resistance of bolted connections. The cavity and cooling link

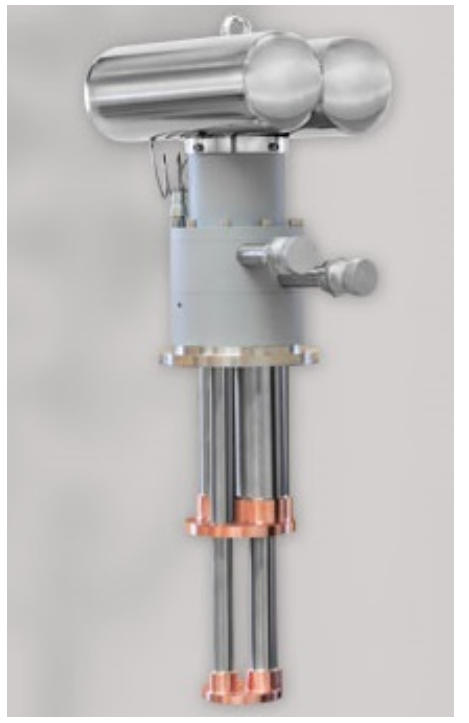

Fig. 3. A Cryomech PT420 Two-Stage Pulse Tube Refrigerator [8].

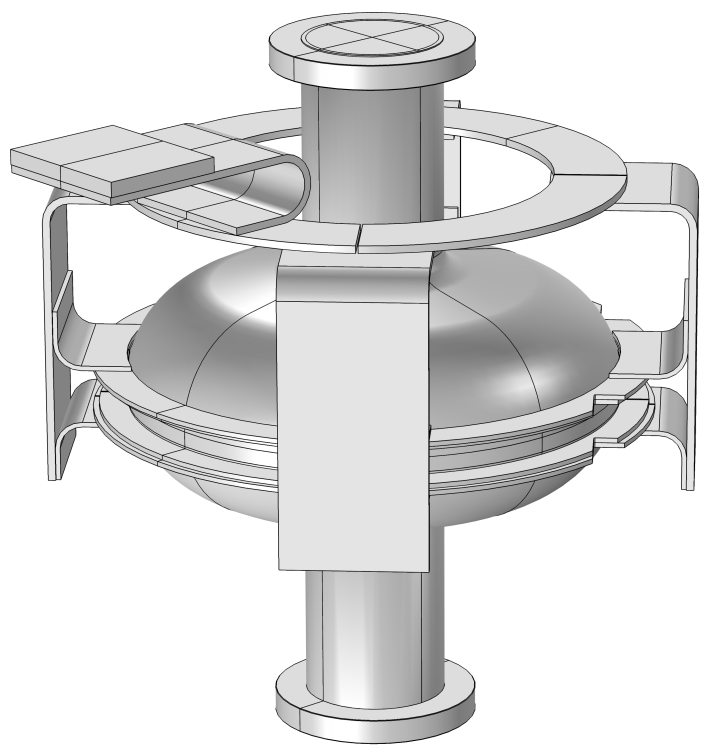

Fig. 4. CAD Model of a $650 \mathrm{MHz}$ SRF Cavity, Cooling Link, and Cryocooler Plate.

are composed of three metals: aluminum, copper, and niobium. In Figure 6, aluminum is shown in blue, copper in orange, and niobium in red.

For a pure, normal conducting metal at cryogenic temperatures, the thermal conductivity $k$ as a function of temperature $T$ can be calculated using the Wiedemann-Franz Law, which relates thermal conductivity to electrical conductivity $\sigma$ [9].

$$
\frac{k}{\sigma}=L_{0} T
$$

The free electron Lorenz number, $L_{0}$, is independent of temperature and material and is calculated from the Boltzmann constant $k_{B}$ and the charge of the electron $e$.

$$
L_{0}=\frac{\pi^{2}}{3}\left(\frac{k_{B}}{e}\right)^{2}=2.45 \times 10^{-8} \mathrm{~W} \Omega \mathrm{K}^{-2}
$$




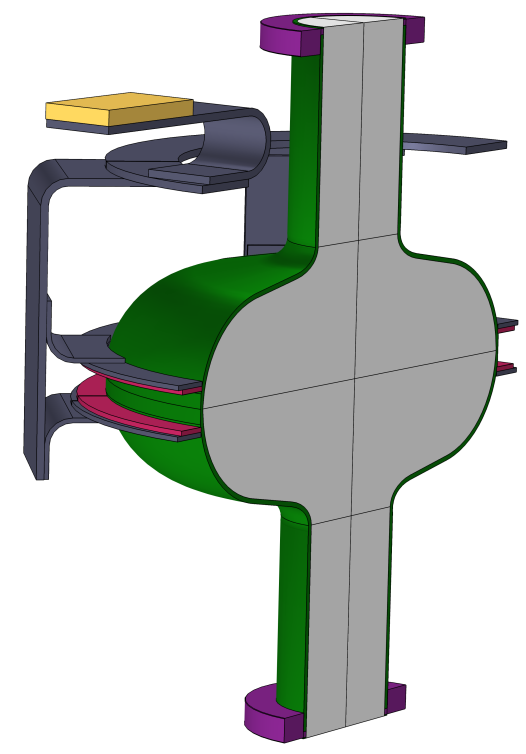

Fig. 5. CAD Model of Cavity (Green), Niobium Rings (Pink), Aluminum Cooling Link (Dark Grey), Cryocooler Plate (Gold), End Caps (Purple), and Vacuum Space Inside Cavity (Light Grey).

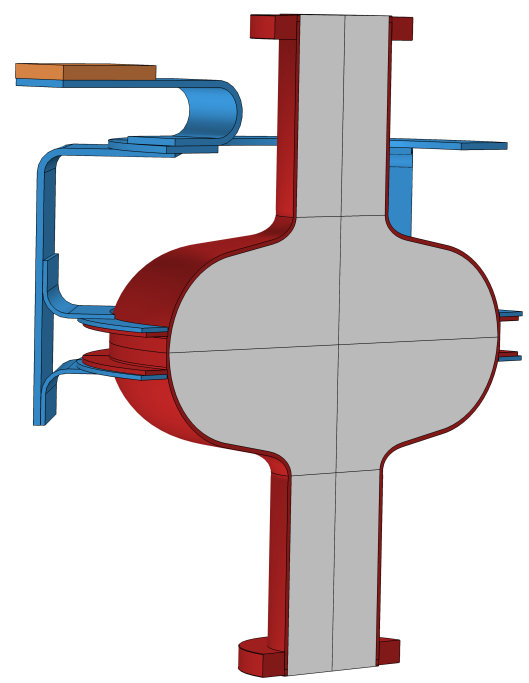

Fig. 6. CAD Model of Geometry Composed of Niobium (red), Aluminum (blue), Copper (orange), and Vacuum (Light Grey).

The residual resistivity ratio (RRR) of a material relates its electrical resistivity at room temperature, $\rho(273 \mathrm{~K})$, to its resistivity at $4.2 \mathrm{~K}, \rho_{0}$.

$$
\mathrm{RRR}=\frac{\rho(273 \mathrm{~K})}{\rho_{0}}
$$

Values of $\rho(273 \mathrm{~K})$ are commonly tabulated, and the conductivity of a material at cryogenic temperatures can be approximated as $\rho_{0}^{-1}$. Combining Equations 8 and 10, the thermal conductivity of a metal at low temperature is

$$
k=L_{0} T \frac{\mathrm{RRR}}{\rho(273 \mathrm{~K})}
$$

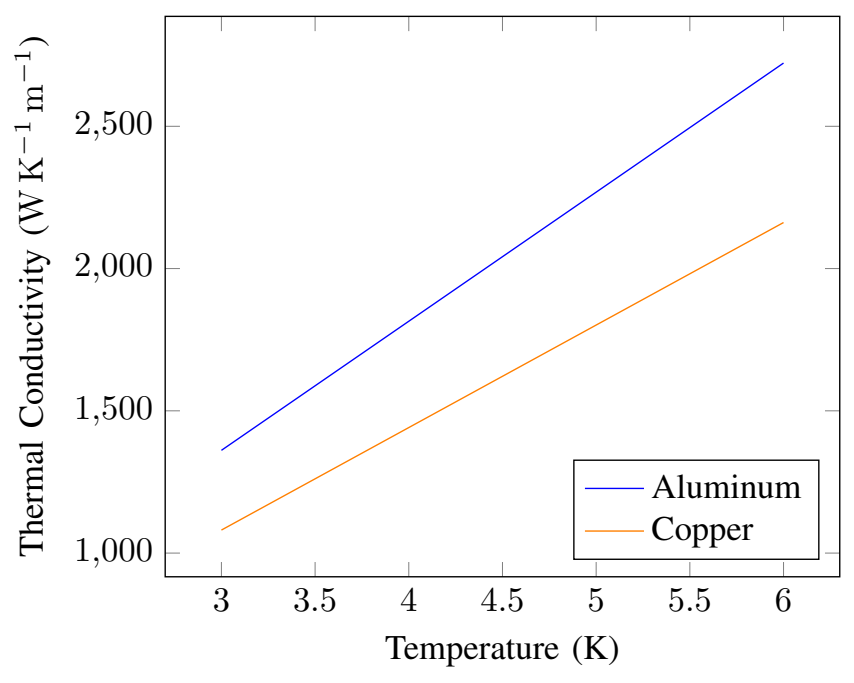

Fig. 7. Thermal Conductivity of 5N Aluminum and 5N Copper [10].

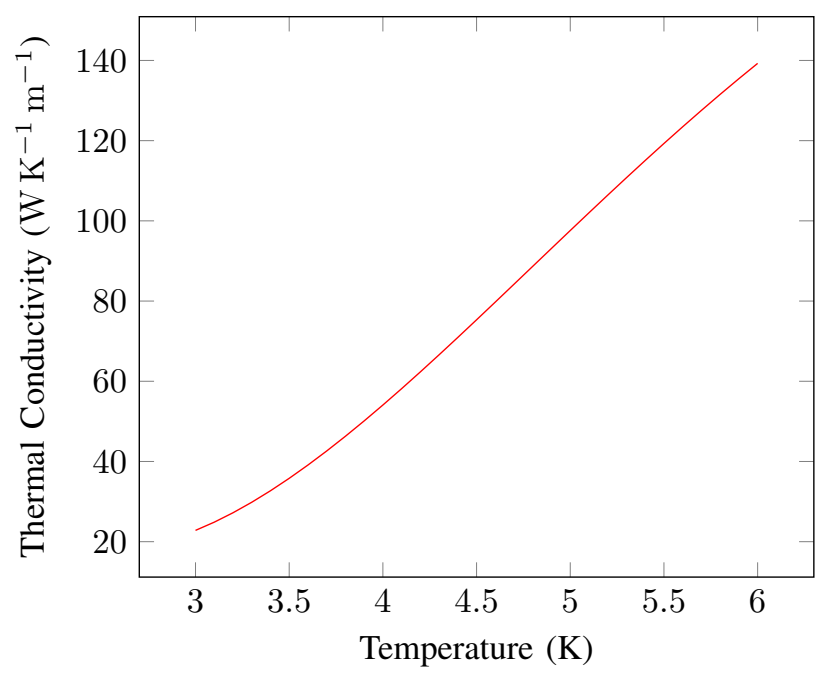

Fig. 8. Thermal Conductivity of Superconducting Niobium [11].

The thermal link is constructed from $5 \mathrm{~N}$ aluminum, aluminum which is at least $99.999 \%$ pure. Values of RRR = 500 and $\rho(273 \mathrm{~K})=2.7 \times 10^{-8} \Omega \mathrm{m}$ were used to find the thermal conductivity of the aluminum. The cryocooler plate is $5 \mathrm{~N}$ copper, which can be modeled with $\mathrm{RRR}=250$ and $\rho(273 \mathrm{~K})=1.7 \times 10^{-8} \Omega \mathrm{m}[10]$. The thermal conductivity of the aluminum and copper are shown in Figure 7.

The SRF cavity and the rings welded around the equator are made of niobium, which is a superconductor with a much more complicated thermal conductivity. The calculations explained by Koechlin and Bonin [11] were used to determine the thermal conductivity of niobium, which is plotted in Figure 8.

To model heat flow through time, the heat capacity of each metal must be known. The NIST Cryogenic Technologies Group Resources maintains an Index of Material Properties [12]. The index provides polynomial curve fits for the specific heat capacity of alloys. The curves for 3003F Aluminum and Copper (OFHC) were used to model $5 \mathrm{~N}$ aluminum and $5 \mathrm{~N}$ 


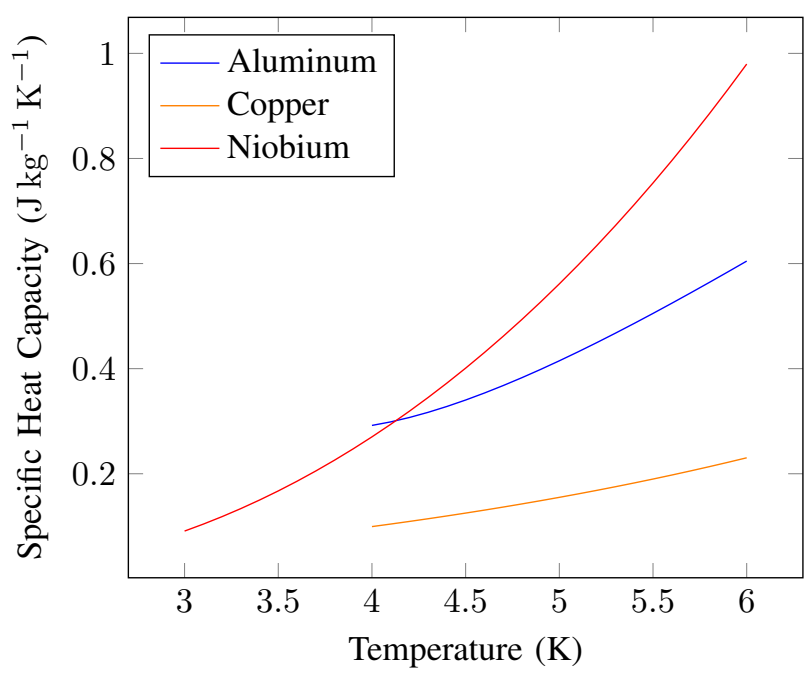

Fig. 9. Heat Capacity of Aluminum, Copper, and Niobium [12], [13].

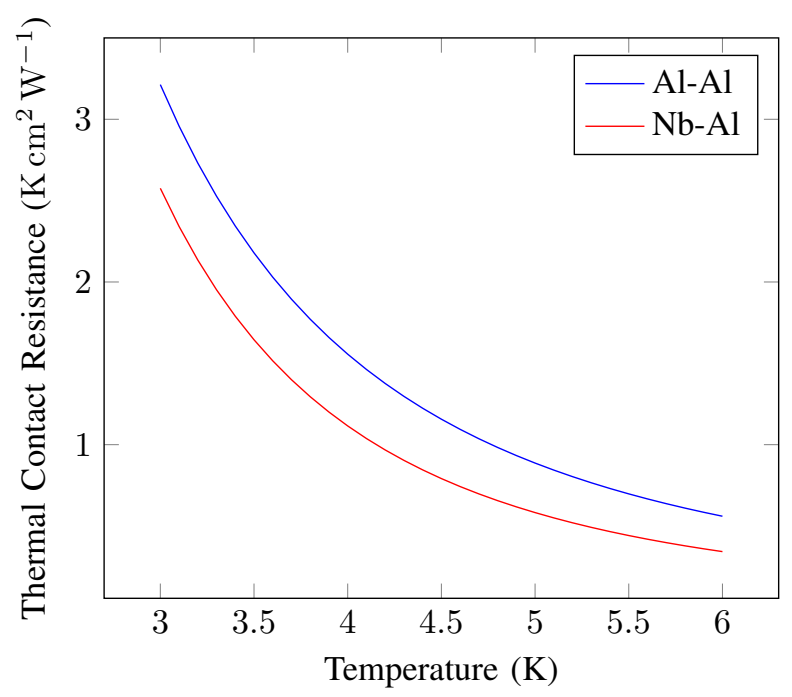

Fig. 10. Thermal Contact Resistance of Bolted $\mathrm{Al}-\mathrm{Al}$ and $\mathrm{Nb}-\mathrm{Al}$ Connections [14].

copper. To model the specific heat capacity of niobium, a polynomial fit published by Andrew Davies for niobium at temperatures less than $9 \mathrm{~K}$ was used [13]. The heat capacities of all three metals are shown in Figure 9.

The thermal contact resistance between bolted connections must also be considered. Using experimental data, the contact resistances between aluminum and niobium and between aluminum and aluminum were found [14]. These contact resistances are shown in Figure 10.

The surface resistance of a $\mathrm{Nb}_{3} \mathrm{Sn}$ coated cavity is another temperature-dependent property and is shown in Figure 11 [15].

To model electromagnetic fields inside the cavity, the space was assumed to be perfect vacuum with the permittivity of free space $\epsilon_{0}$ and the permeability of free space $\mu_{0}$.

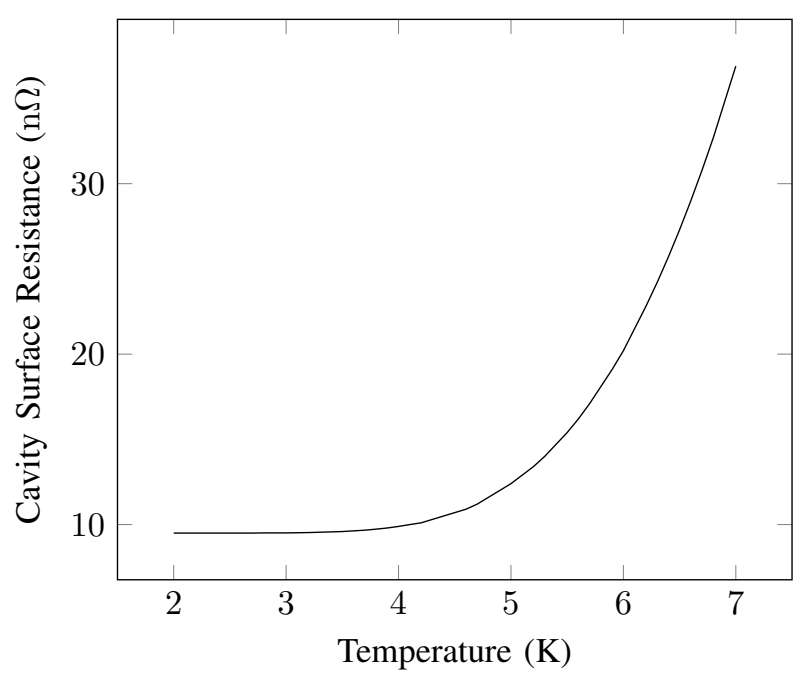

Fig. 11. Surface Resistance of SRF Cavity with $\mathrm{Nb}_{3} \mathrm{Sn}$ Coating (courtesy: Sam Posen).

\section{Cryocooler Heat Capacity Measurements}

The heat capacity of the cryocooler describes the amount of dissipated power it can absorb from the cavity at a given temperature. Accurate measurements of the cryocooler's heat capacity are crucial for simulating heat transfer through the system.

To measure the heat capacity, a thermometer and a cartridge heater, which uses a resistive load to produce Joule heating, were connected to each stage of the pulse tube refrigerator. A Lakeshore TC 355 temperature controller with Labview was used to set the output of the first stage heater, H1, and read the temperature of both stages. Because the temperature controller can set the heater output with only limited precision, the heater on the second stage, $\mathrm{H} 2$, was powered more precisely with a Keithley 2230G-30-1 current source. As the current was varied, the voltage across the heater was measured using a Keysight 33461 A digital multimeter, and the input power to the heater was found by Equation 12 .

$$
P_{\text {in }}=I V
$$

The power supplied to $\mathrm{H} 1$ was adjusted to five values between $0-75 \mathrm{~W}$, and the power supplied to $\mathrm{H} 2$ was varied between $0-5 \mathrm{~W}$. For each case, the temperature of stage 2 (T2) was recorded, along with the amplitude of the cyclic temperature oscillations. Figure 12 shows the temperature of stage 2 as a function of the input power to $\mathrm{H} 1$ and $\mathrm{H} 2$. Note that increasing the heat load on stage $2(\mathrm{H} 2)$ clearly increases the temperature, while the heat load on stage $1(\mathrm{H} 1)$ has only a limited impact on the temperature. Therefore, the main factor influencing the cryocooler temperature is the dissipated power from the SRF cavity, not the temperature of stage 1. Figure 13 shows the amplitude of the cyclic temperature oscillations at stage 2 for various input power. The period of oscillation is about one second, and the amplitude of these oscillations also increases with the input power. 


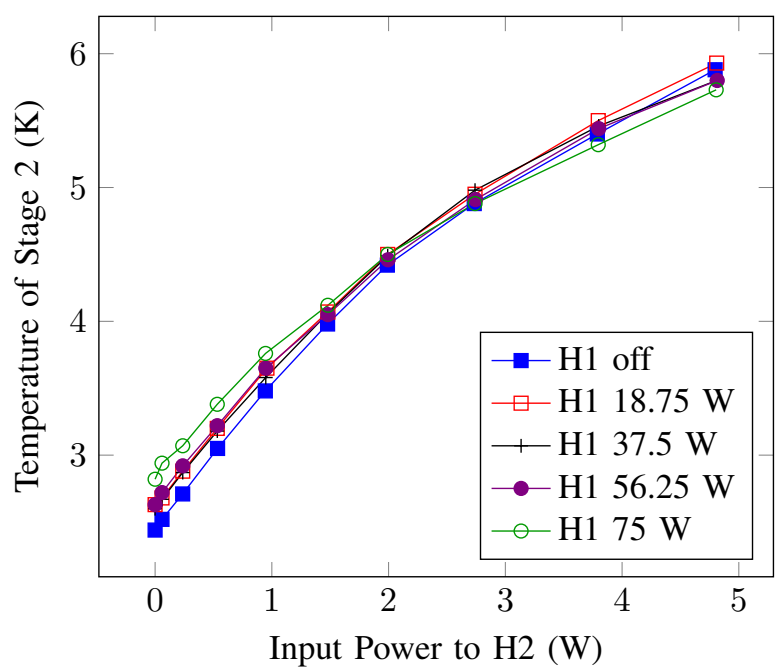

Fig. 12. Temperature at Stage 2 for Input Powers H1 and H2.

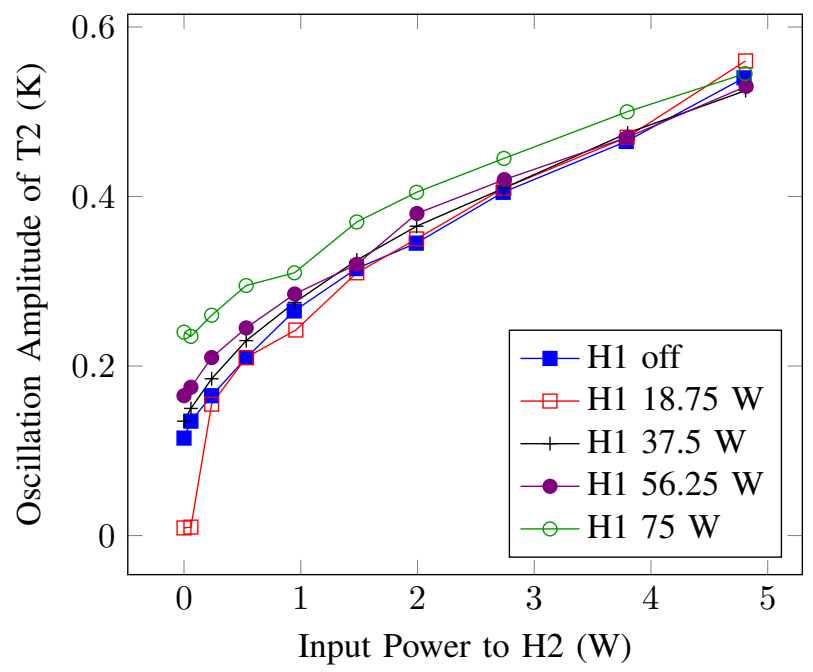

Fig. 13. Amplitude of Temperature Oscillations at Stage 2 for Input Powers $\mathrm{H} 1$ and $\mathrm{H} 2$.

\section{Simulation Procedure}

The final simulation goals were accomplished by splitting the work into discrete steps, which provided several advantages. The simulations were simpler to set up and faster to run, and the results were much easier to validate. Dividing the work into smaller parts also required less computing power, less memory, and fewer software licenses.

1) Heat Transfer Through Cooling Link: First, heat transfer through the cryocooler plate, cooling link, and niobium rings was modeled. The geometry used is shown in Figure 14. The dissipated power of the cavity, $P_{d}$, was represented as a heat flux entering the cooling link through the weld faces of the niobium rings. The temperature at the top of the cryocooler plate, $T_{\text {cryo }}$, was determined for the given heat flux from Figure 12. Using these two boundary conditions, a heat transfer simulation was performed to find the equilibrium temperature across the geometry. The input parameter $P_{d}$ was varied, and the average temperature of the weld faces, $T_{\text {weld }}$,

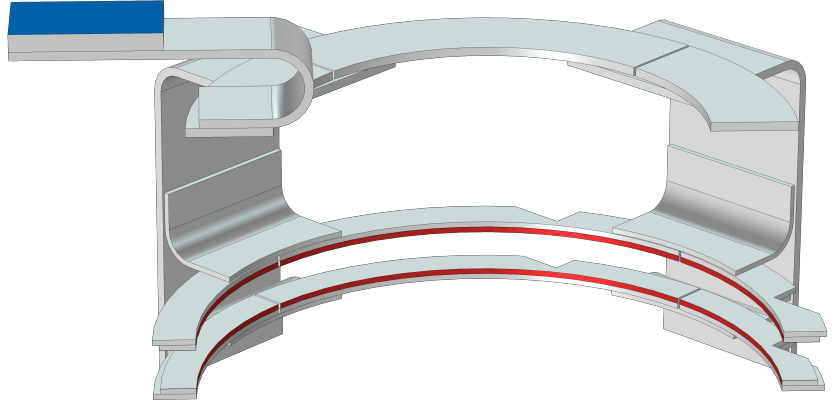

Fig. 14. Geometry Used for First Heat Transfer Simulation with Weld Faces (red) and Top of Cryocooler Plate (blue).

was recorded for each case.

2) Cryocooler Oscillations: The previous simulation focused on finding the steady state thermodynamic solution of the cooling link. However, the cryocooler temperature fluctuates periodically, so assuming a constant temperature at the top of the cryocooler plate is unrealistic. To model the cyclic variation in temperature, the temperature at the cryocooler plate was modified to a periodic function.

$$
T(t)=T_{0}+A \sin (2 \pi f t)
$$

Here $T_{0}$ is the cryocooler's average temperature, $A$ is the amplitude of oscillation, and $f$ is the frequency of oscillation. For the simulation, values of $T_{0}=4.2 \mathrm{~K}, A=0.25 \mathrm{~K}$, and $f=$ $1 \mathrm{~Hz}$, which model the cryocooler operation with a dissipated power of about $1.5 \mathrm{~W}$ (see Figures 12, 13), were used.

Due to time constraints, the time-dependent simulation was performed only on the partial geometry in Figure 14, not on the full geometry. The oscillations in temperature at the weld faces of the rings were compared to the temperature oscillations at the cryocooler plate.

3) Multiphysics Simulation of Cavity: In the second set of simulations, the cavity was simulated without the niobium rings and cooling link. An eigenfrequency study was performed on the vacuum space within the cavity to find the resonant frequency $\omega$, along with the $H$ field, $G, U, R / Q$, and $B_{p k}$. The simulation returns relative values for the energy levels within the cavity, but without information about the driving source, it cannot calculate the absolute magnitude of the electric and magnetic fields. To scale the results, at least one value must be assumed. For this simulation, $E_{a c c}$ was provided as a parameter, and the electric and magnetic field values were multiplied by the ratio of $E_{a c c}$ to the arbitrary accelerating gradient found in the simulation to produce meaningful values.

The magnetic field $H$ on the inner surface of the cavity was then used as input to a heat transfer simulation through 


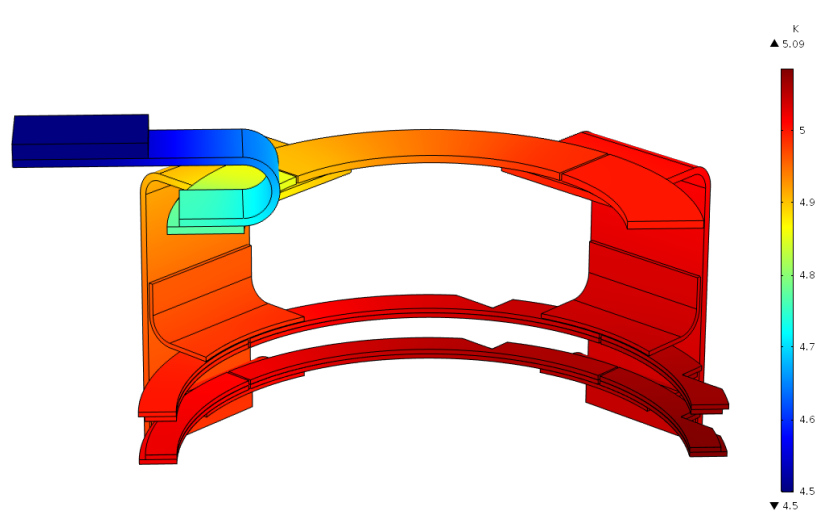

Fig. 15. Temperature Distribution Through Cooling Link with $P_{d}=2 \mathrm{~W}$ and $T_{\text {cryo }}=4.5 \mathrm{~K}$.

the cavity. The dissipated power in each mesh element, $d A$, was calculated using the known $H$ field and the temperaturedependent $R_{s}$. The heat flows out through the notches in the cavity where the niobium rings are welded on. These notches were approximated as having a spatially uniform, constant temperature $T_{n}$, which was found from the accelerating gradient and the results of the thermal link simulation. The cavity simulation was repeated for different values of $E_{a c c}$, and temperature difference across the cavity $\Delta T_{\text {cavity }}$ was recorded for each case.

Additionally, the effects of $R_{s}$ on $\Delta T_{\text {cavity }}$ were examined. The $R_{s}$ values shown in Figure 11 assume an even coating of $\mathrm{Nb}_{3} \mathrm{Sn}$ across the cavity. The simulation was repeated for cases where the coating was properly applied on only $90 \%$ and on only $80 \%$ of the cavity. To model these cases, the surface resistance of a "bad" coating, $R_{s, b a d}$ was calculated as three times the ideal surface resistance, $R_{s, \text { ideal }}$, which is shown in Figure 11. The total surface resistance of a cavity with a coating properly applied on pct percent of the surface was then found as

$$
R_{s}=p c t R_{s, i d e a l}+(1-p c t) R_{s, b a d}
$$

In addition, a pure niobium cavity with no $\mathrm{Nb}_{3} \mathrm{Sn}$ coating was simulated, and the limitations of this cavity were examined.

4) Multiphysics Simulation of Entire Geometry: Finally, the entire geometry was simulated. The electromagnetic fields within the cavity were calculated, and the heat flow of the dissipated power from the inner surface of the cavity to the cryocooler plate was modeled. The results from this simulation support the previous simulations.

\section{Simulation Results}

\section{A. Heat Transfer Through Cooling Link}

The temperature distribution of the cooling link was found for eighteen values of the dissipated power $P_{d}$. Shown in Figure 15 is the temperature map for $P_{d}=2 \mathrm{~W}$. Figure 16 shows the average temperature of the niobium weld faces as a function of dissipated power.

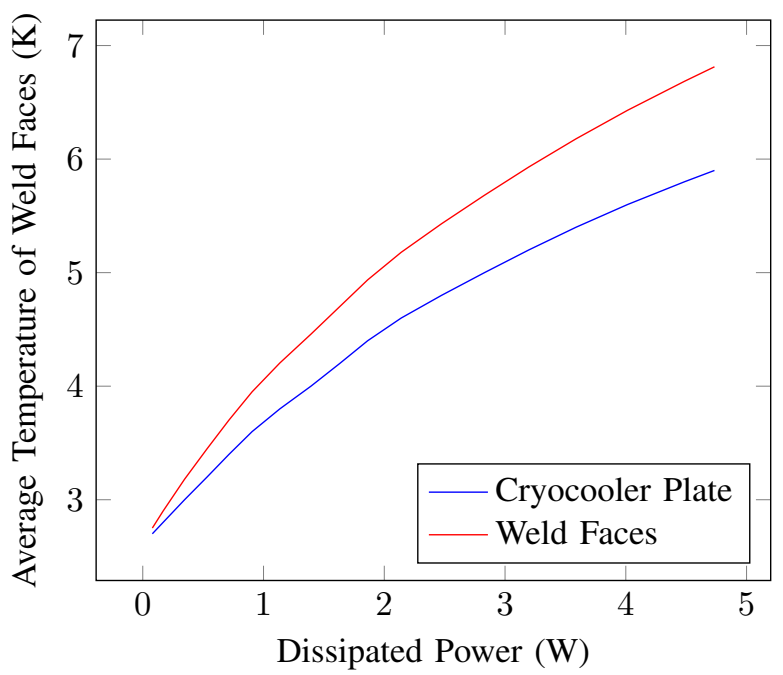

Fig. 16. Average Temperature of Cryocooler Plate and Niobium Ring Weld Faces For Various Total Dissipated Power.

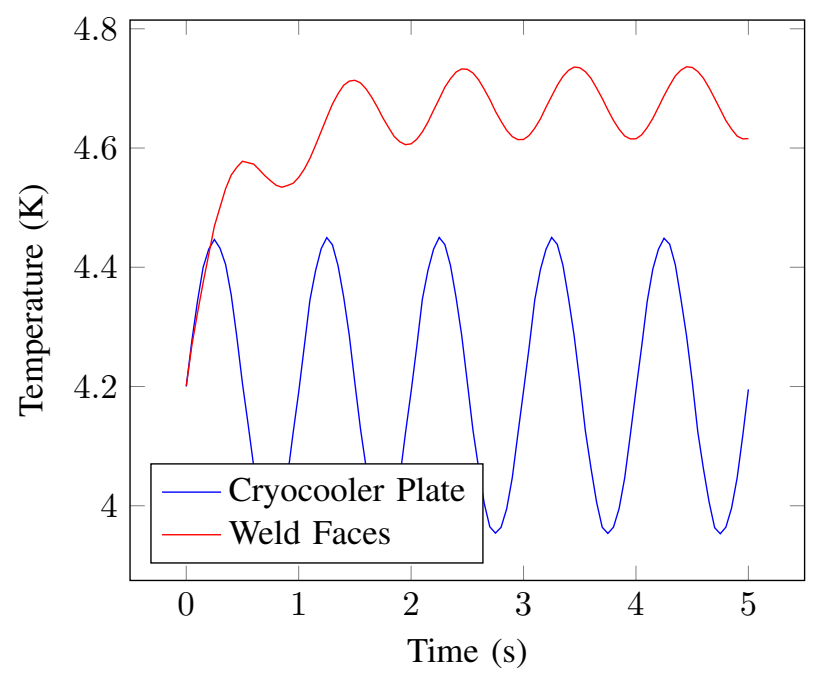

Fig. 17. Temperature Oscillations with Time.

\section{B. Cryocooler Oscillations}

The periodic cryocooler temperature, approximated as a sine wave, is shown in blue in Figure 17, and the spatial average of the weld face temperature is shown in red. Although the temperature at the weld faces does oscillate with time, the amplitude of the oscillations is about one-fourth of the amplitude of the cryocooler oscillations. Since niobium is a relatively poor thermal conductor, these oscillations would likely attenuate even further across the cavity surface.

\section{Multiphysics Simulation of Cavity}

The magnitude of the electric and magnetic fields inside the cavity were found in the second simulation and are shown, scaled for $E_{a c c}=10 \mathrm{MV} / \mathrm{m}$, in Figures 18 and 19. The electric field is strongest in the center of the cavity and points along the cavity axis. The magnetic flux is strongest around the cavity equator. The calculated resonant frequency of the cavity is 647 


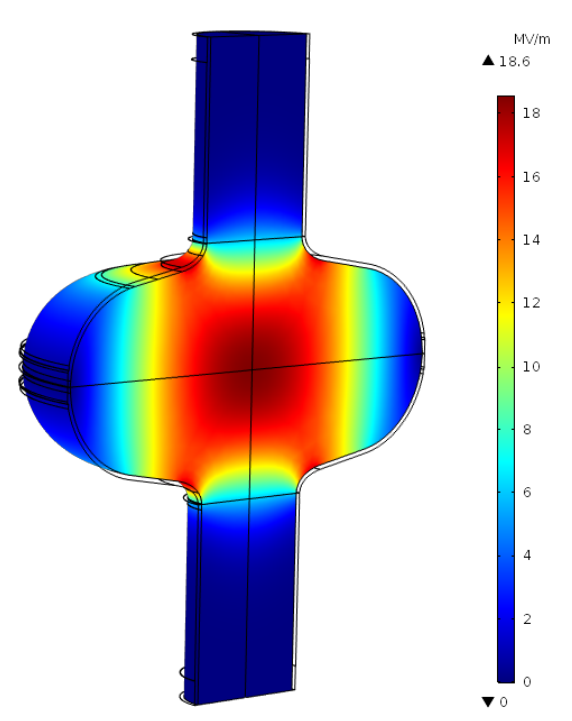

Fig. 18. Electric Field Magnitude Inside the Cavity.

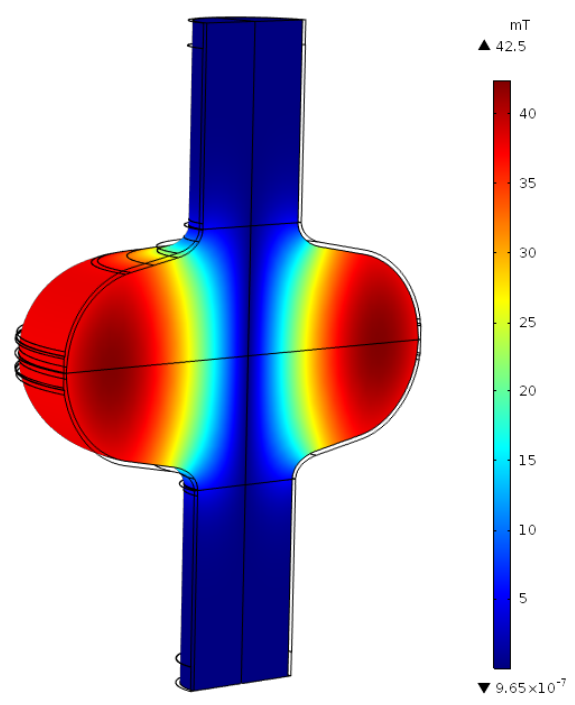

Fig. 19. Magnetic Flux Magnitude Inside the Cavity.

MHz. Using the frequency and fields, we can find the other $\mathrm{RF}$ parameters and compare with the theoretical values for this shape cavity. The RF parameters found with an assumed accelerating gradient of $10 \mathrm{MV} / \mathrm{m}$ are shown in Table I.

Using the magnetic field calculated in the RF eigenfrequency study, the temperature distribution across the cavity was found. The heat flow depends on the choice of $E_{a c c}$ and $R_{s}$. Figure 20 shows an example of the temperature across the cavity with $E_{a c c}=10 \mathrm{MV} / \mathrm{m}, T_{n}=4.52 \mathrm{~K}$, and $100 \%$ ideal $R_{s}$. The temperature difference across the elliptical surface of the cavity is $130 \mathrm{mK}$, which is small compared to the temperature difference across the thermal link. Moreover, the average temperature and maximum temperature of the cavity elliptical surface are within $2 \%$ of each other. These trends were also apparent for the other simulated values of

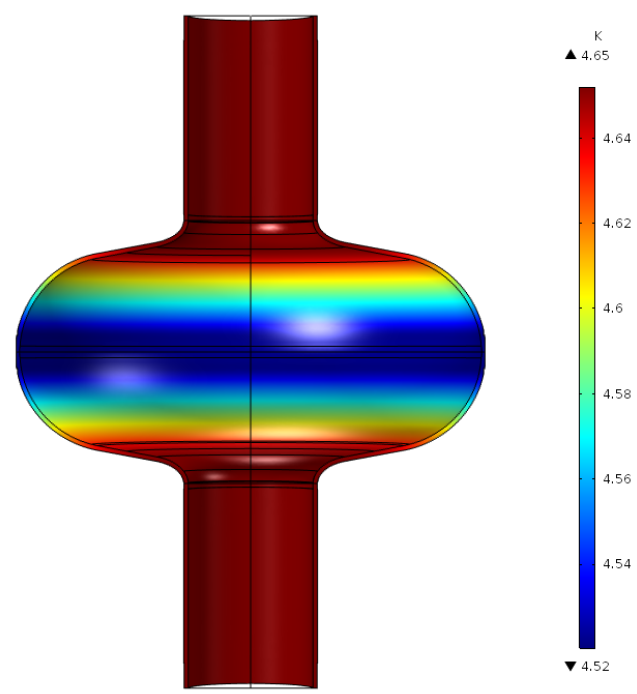

Fig. 20. Temperature Distribution in Cavity.

the accelerating gradient and surface resistance. Therefore, the spatial temperature variations across the cavity are largely inconsequential, and only the average temperature of the cavity elliptical surface is reported for the remainder of the simulation results.

The accelerating gradient was adjusted to four different values. For each value of $E_{a c c}$, the dissipated power was approximated using Equation 7, using the simulated values for the geometric factor and normalized shunt impedance shown in Table I and assuming a surface resistance of $10 \mathrm{n} \Omega$. The notch temperature $T_{n}$ was then determined from Figure 16. Using this notch temperature and the known $H$ field on the cavity surface, heat flow through the cavity was modeled. The accelerating gradient and surface resistance were varied, and the average temperature of the cavity was recorded. These results are shown in Figure 21. It can be seen that small changes in the quality of the $\mathrm{Nb}_{3} \mathrm{Sn}$ coating make very little impact on the cavity temperature.

To investigate the importance of the $\mathrm{Nb}_{3} \mathrm{Sn}$ coating, a cavity without the coating was simulated. A notch temperature $T_{n}=$ $5 \mathrm{~K}$ and a dissipated power $P_{d}=2 \mathrm{~W}$ were assumed. At $5 \mathrm{~K}$, pure, uncoated niobium has an approximate surface resistance of $680 \mathrm{n} \Omega$ [16]. Using Equation 7, the accelerating gradient for these conditions was found to be $1.4 \mathrm{MV} / \mathrm{m}$. The simulated temperature distribution for this case was nearly identical to that shown in Figure 20 with a maximum temperature of $5.12 \mathrm{~K}$. However, the surface resistance of pure niobium rises with temperature, and the heat capacity of the cryocooler is quickly exceeded at accelerating gradients above $1.4 \mathrm{MV} / \mathrm{m}$. $\mathrm{The} \mathrm{Nb}_{3} \mathrm{Sn}$ coating is a key factor in enabling conduction cooling for cavities at mid-range accelerating gradients.

\section{Multiphysics Simulation of Entire Geometry}

The previous simulations can be used together to approximate the temperature across the cavity and cooling link for different values of the accelerating gradient. To verify these 
TABLE I

Simulated RF PARAMETERS AND THEORETICAL VALUES.

\begin{tabular}{c|c|c|c} 
Parameter & Symbol & Simulated Value & Theoretical Value \\
\hline Peak Surface Magnetic Flux & $B_{p k}$ & $38.2 \mathrm{mT}$ & $36.0 \mathrm{mT}$ \\
Peak Surface Electric Field & $E_{p k}$ & $17.5 \mathrm{MV} / \mathrm{m}$ & $16.0 \mathrm{MV} / \mathrm{m}$ \\
Geometric Factor & $G$ & 265 & 266 \\
Normalized Shunt Impedance & $R / Q$ & 138 & 138 \\
Dissipated Power & $P_{d}$ & $1.50 \mathrm{~W}$ & \\
Stored Energy & $U$ & $9.49 \mathrm{~J}$ & \\
Quality Factor & $Q_{0}$ & $2.6 \times 10^{10}$ &
\end{tabular}

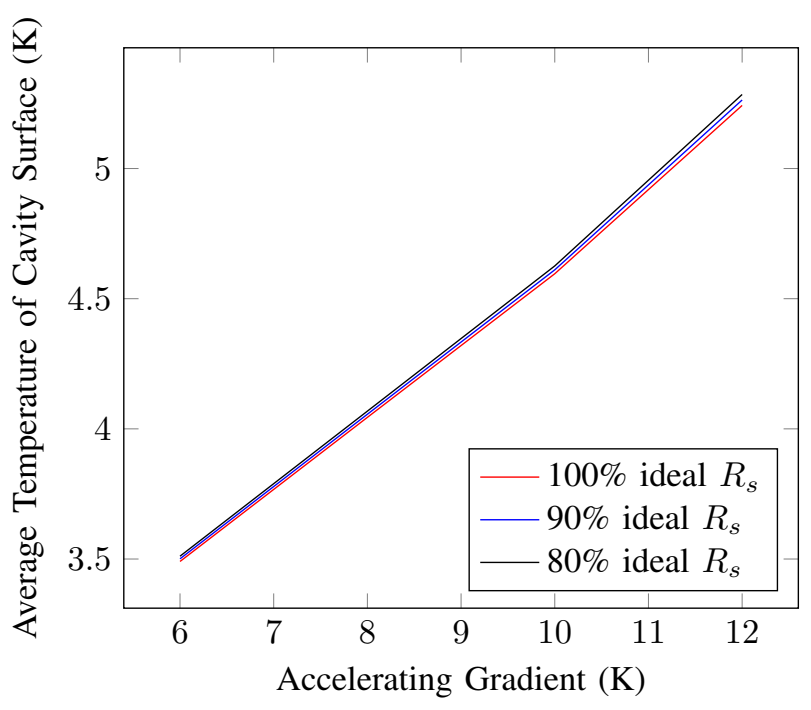

Fig. 21. Average Cavity Temperature for Varying Accelerating Gradient and Surface Resistance.

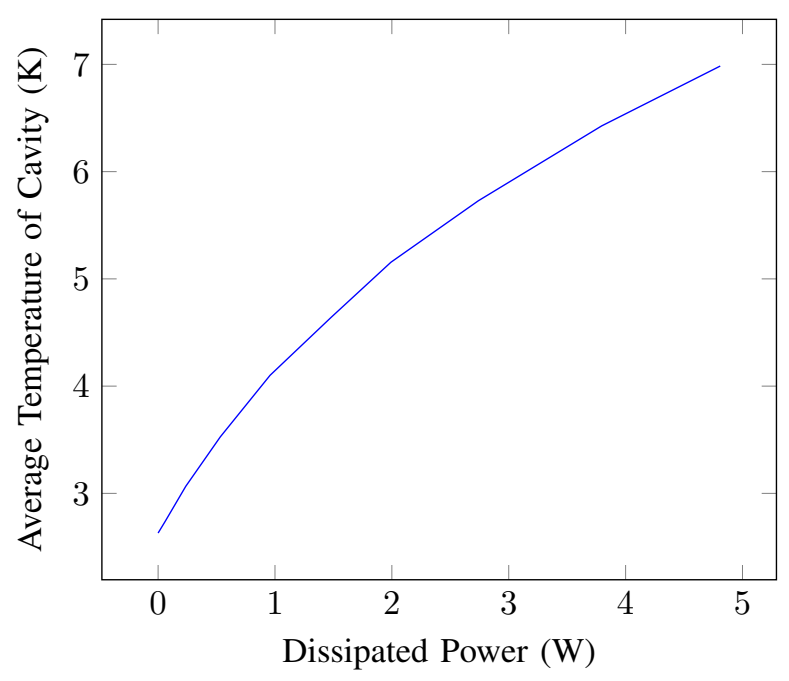

Fig. 22. Average Cavity Temperature for Varying Dissipated Power.

approximations, a multiphysics simulation of the entire geometry was performed. The maximum temperature of the niobium, $T_{\text {max }}$, was found as a function of dissipated power, $P_{d}$. The results are shown in Figure 22. These results match very well with the combined results of the previous simulations.

\section{Conclusions}

Conduction cooling of a $\mathrm{Nb}_{3} \mathrm{Sn}$ coated single cell $650 \mathrm{MHz}$ SRF cavity was simulated using the geometry of the cavity, measured heat capacity of a Cryomech PT420 cryocooler, and thermodynamic properties of a designed cooling link. For accelerating gradients between 6 and $12 \mathrm{MV} / \mathrm{m}$, the average equilibrium temperature of the cavity was below 6 $\mathrm{K}$, within the superconducting regime of niobium. Moreover, the temperature of the cavity elliptical surface was found to be nearly spatially uniform. The $\mathrm{Nb}_{3} \mathrm{Sn}$ coating was shown to be of critical importance for operating the cavity at an accelerating gradient above $1.5 \mathrm{MV} / \mathrm{m}$. Additionally, the temperature oscillations of the cryocooler were modeled, and the amplitude of temperature fluctuations significantly dampened at the location of the cavity. Based on the simulation results, a commercial cryocooler and the designed thermal link are a viable alternative to liquid helium for SRF cavities with $\mathrm{Nb}_{3} \mathrm{Sn}$ coating in applications which require an accelerating gradient of $12 \mathrm{MV} / \mathrm{m}$ or below.

\section{ACKNOWLEDGEMENTS}

This manuscript has been authored by Fermi Research Alliance, LLC under Contract No. DE-AC02-07CH11359 with the U.S. Department of Energy, Office of Science, Office of High Energy Physics. Special thanks to Ivan Gonin, Michael Geelhoed, Charles Thangaraj, Oleg Prokofiev, Andrew Dalesandro, Thomas Kroc, Andrei Lunin, Aaron Sauers, and the SIST internship committee.

\section{REFERENCES}

[1] R. W. Hamm and M. E. Hamm, "The beam business: Accelerators in industry," Phys. Today, vol. 64N6, pp. 46-51, 2011.

[2] W. Martienssen and H. Warlimont, Springer handbook of condensed matter and materials data. Springer Science \& Business Media, 2006.

[3] M. Tinkham, Introduction to superconductivity. Courier Corporation, 2004.

[4] B. Barish and J. E. Brau, "The International Linear Collider," Int. J. Mod. Phys., vol. A28, no. 27, p. 1330039, 2013.

[5] R. Radebaugh, "Pulse tube cryocoolers for cooling infrared sensors," in Infrared Technology and Applications XXVI, vol. 4130, pp. 363-380, International Society for Optics and Photonics, 2000.

[6] R. F. Barron, Cryogenic systems. Clarendon Press, 1985.

[7] A. De Waele, "Basic operation of cryocoolers and related thermal machines," Journal of Low Temperature Physics, vol. 164, no. 5-6, p. $179,2011$.

[8] Cryomech PT420. http://www.cryomech.com/cryorefrigerators/ pulse-tube/pt420/. Accessed 2018-07-25.

[9] S. W. Van Sciver, Helium cryogenics, ch. 2 Low-Temperature Materials Properties. Springer Science \& Business Media, 2012.

[10] A. L. Woodcraft, "Recommended values for the thermal conductivity of aluminium of different purities in the cryogenic to room temperature range, and a comparison with copper," Cryogenics, vol. 45, no. 9, pp. 626-636, 2005. 
[11] F. Koechlin and B. Bonin, "Parametrization of the niobium thermal conductivity in the superconducting state," Superconductor Science and Technology, vol. 9, no. 6, p. 453, 1996.

[12] P. Bradley, "Index of material properties," NIST Cryogenic Technology Resources Website. Accessed 2018-07-23.

[13] A. Davies, "Material properties data for heat transfer modeling in nb3sn magnets," Illinois Accelerator Institute, vol. 17, pp. 1-53, 2011.

[14] R. Dhuley, M. Geelhoed, and J. Thangaraj, "Thermal resistance of pressed contacts of aluminum and niobium at liquid helium temperatures," Cryogenics, vol. 93, pp. 86-93, 2018.

[15] S. Posen. Fermi National Accelerator Laboratory. Private Communication.

[16] M. Liepe, "SRIMP." https://www.classe.cornell.edu/ liepe/webpage/ researchsrimp.html. Accessed 2018-08-02. 